\title{
$\mathrm{PI}$ 관측기의 불확실성 추정을 이용한 표면부착형 영구자석 동기기의 전류 제어기 설계
}

\begin{tabular}{|c|}
\hline 논 문 \\
\hline $60-5-17$ \\
\hline
\end{tabular}

\section{Design of a Surface-Mounted PMSM Current Controller Using Uncertainty Estimation with a PI Observer}

\author{
김 인 혁**최 대 식** 손 영 익 ${ }^{\dagger}$
}

(In Hyuk Kim· Dae Sik Choi · Young Ik Son)

\begin{abstract}
This paper presents a robust current controller for a surface-mounted permanent magnet synchronous motor(SPMSM) by using a PI observer. The decoupling PI(proportional-integral) controller combined with an additional feed-forward compensation has been used for the current controller. The classical feed-forward compensation using velocity information and system parameters is not expected to achieve a robust performance against parameter uncertainties. This paper has adopted a PI observer for the feed-forward compensation to cope with parameter uncertainties without using velocity information. A simple PI observer has been designed to compensate the disturbances that represent velocity coupled terms and parameter uncertainties. Experimental results as well as computer simulations with 630W SPMSM confirm that the proposed approach can deal with the effects of the disturbance and improve the control performance.
\end{abstract}

Key Words : SPMSM, Current control, Feed-forward compensation, PI observer

\section{1. 서 론}

영구자석 동기기(Permanent Magnet Synchronous Motor, $\mathrm{PMSM})$ 는 넓은 운전 영역에 걸쳐서 높은 효율을 유지하는 특성으로 인해 고효율 운전이 요구되는 응용 분야에 많이 사용되고 있으며 그 중에서도 표면부착형 영구자석 동기기 (Surface-Mounted PMSM, SPMSM)는 비교적 제어가 간단 하여 많은 적용 분야에서 연구되고 있다[1-4].

전동기 구동을 위한 토크는 전기자 전류에 비례하고, 전동 기의 속도 제어를 위한 순차(cascade) 제어 기법에서도 전 류를 먼저 제어해야 하는 등 전동기의 전류 제어 문제는 다 양한 적용 분야에서 다뤄지고 있다[5-9]. 본 논문에서는 $\mathrm{SPMSM}$ 의 전류 제어기 설계 문제를 다룬다.

SPMSM의 전류 제어 기법으로 주제어기인 PI 제어기에 추가로 전기자 반작용 및 역기전력 항을 외란으로 고려하여 전향(feed-forward; $\mathrm{FF}$ ) 보상을 함께 사용하는 방법 (Technical Optimum Method)이 많이 활용되고 있다[9]. 이 제어 기법은 전류 방정식의 속도관련 항을 소거하여 기계 방정식과 분리(decoupling)함으로써 PI 제어기로 폐루프 시 스템을 간단하게 제어할 수 있다는 장점이 있다. 이 때, 일 반적으로 속도 정보와 시스템 파라미터를 사용하여 전기자 반작용 및 역기전력 항을 직접 보상에 이용한다[9-11]. 이와 같은 경우 전동기의 공칭 파라미터를 사용하게 되므로 속도

* 정 회 원 : (주)한국미쓰비시엘리베이터 전기개발팀

** 준 회 원 : 명지대 공대 전기공학과 석사과정

† 교신저자, 정회원 : 명지대 공대 전기공학과 부교수

E-mail : sonyi@mju.ac.kr

접수일자 : 2011년 1월 31일

최종완료 : 2011년 3월 30일
추정 오차, 파라미터 변화, 모델링 오차와 같은 불확실성에 대해 전류 제어 성능이 저하될 수 있다.

본 논문에서는 전향 보상에 이용하는 항에 존재하는 불확 실성에 강인한 제어기를 설계하기 위한 방법으로 PI 관측기 를 전향 보상기로 사용한다[12]. 제안된 방법은 불확실성을 상수로 가정하고 간단한 PI 관측기를 통해 불확실성을 추정 하여 제어 입력에 보상하는 형태이므로 실제 시스템에 쉽게 적용할 수 있다.

시스템 불확실성에 대처하기 위한 다른 방법으로 $H_{\infty}$-제 어기 등 다양한 고급 제어 알고리즘이 제안되었으나 그 복 잡성과 설계 과정에서 결정해야하는 제어 변수가 증가하는 등 실제적인 응용 분야에서 적합하지 않은 경우가 존재한다 [7]. 본 논문에서 제안하는 방법은 구조가 간단하고 디지털 제어기로도 쉽게 구현할 수 있어 전류 제어와 같은 실제적 인 전동기 제어 문제에 편리하게 활용될 수 있다.

본 논문의 구성은 다음과 같다. 2.1절과 2.2절에서는 시스 템의 모델 식 및 일반적인 전향 보상 방법을 소개하고, 2.3 절에서는 PI 관측기의 불확실성 추정을 이용하는 전류 제어 기를 제안한다. 3.1절에서는 모의실험을 통해 제안된 방법을 $\mathrm{SPMSM}$ 의 불확실성 추정에 적용한 결과를 분석하고, 3.2 절 에서는 $630 \mathrm{~W}$ SPMSM를 대상으로 실제 실험을 통해 디지 털 제어기로 구현된 제안된 알고리즘의 적용 가능성을 확인 한다.

\section{2. 본 론}

\section{$2.1 \mathrm{SPMSM}$ 의 동기 좌표계 모델}

표면부착형 영구자석 동기기(SPMSM)의 수학적 모델을 위해 회전자의 전기 각속도 $\omega_{r}$ 로 회전하는 $\mathrm{d}-\mathrm{q}$ 동기 좌표계 가 사용된다[9,13]. d-q 변환을 사용하면 3상 교류 전압 및 
전류가 동기 좌표계의 직류 전압 $\left(v_{d}^{r}, v_{q}^{r}\right)$ 과 직류 전류 $\left(i_{d}^{r}\right.$, $\left.i_{q}^{r}\right)$ 로 변환되고 전동기의 모델 식은 다음과 같다[9,13].

$$
\begin{gathered}
v_{d}^{r}=R_{s} i_{d}^{r}+L_{s} \frac{d i_{d}^{r}}{d t}-\omega_{r} L_{s} i_{q}^{r} \\
v_{q}^{r}=R_{s} i_{q}^{r}+L_{s} \frac{d i_{q}^{r}}{d t}+\omega_{r}\left(L_{s} i_{d}^{r}+\Lambda_{m}\right) \\
\tau_{e}=\frac{3}{2} P \Lambda_{m} i_{q}^{r}=J \frac{d \omega_{m}}{d t}+B \omega_{m}+T_{L}, \omega_{m}=\omega_{r} / P
\end{gathered}
$$

위 식에서 $R_{s}, L_{s}$ 는 고정자의 저항과 인덕턴스이고 $\Lambda_{m}$ 은 영 구자석의 쇄교 자속을 나타낸다. 그리고 $\omega_{m}$ 은 전동기의 기 계 각속도이며 $P, J, B, T_{L}$ 는 각각 모터의 극 짝수, 관성 질 량, 마찰 계수, 부하 토크를 나타낸다.

식 (1)-(2)는 SPMSM의 전기 모델식이고, 식 (3)은 모델 의 기계식이라 부른다. 전류에 관한 식 (1)-(2)는 기계식 (3) 과 전기 각속도 $\omega_{r}$ 을 통해 서로 결합되어(coupled) 있음을 볼 수 있다. 또한, 식 (3)에서 $\mathrm{q}$ 축 전류 $i_{q}^{r}$ 에 의해 전동기 구 동을 위한 토크 $\tau_{e}$ 가 발생함을 알 수 있다.

\section{2 전향보상 $+\mathrm{Pl}$ 전류 제어기}

SPMSM의 전류 제어를 위해 전체 모델 식 (1) (3)을 사 용하는 대신 식 (1)-(2)를 대상으로 그 중 각속도 $\omega_{r}$ 이 곱해 진 전기자 반작용 및 역기전력 항을 외란으로 고려하는 방 법이 $\mathrm{AC}$ 전동기 분야에서 많이 사용되고 있다[9]. 즉, 아래 식 (4)-(5)와 같이 식 (1)-(2)에서 $\omega_{r}$ 이 곱해진 항은 외란으 로 보고 전향 보상을 통해 분리된(decoupled) 두 1차 시스템 에 대한 PI 제어기를 설계하는 방법이다.

$$
\begin{aligned}
e_{1} & =i_{d}^{r^{*}}-i_{d}^{r} \\
v_{d}^{r} & =K_{p} e_{1}+K_{i} \int e_{1} d t-\omega_{r} \hat{L}_{s} i_{q}^{r} \\
e_{2} & =i_{q}^{r^{*}}-i_{q}^{r} \\
v_{q}^{r} & =K_{p} e_{2}+K_{i} \int e_{2} d t+\omega_{r}\left(\hat{L}_{s} i_{d}^{r}+\hat{\Lambda}_{m}\right)
\end{aligned}
$$

여기서 $i_{d}^{r *}, i_{q}^{r *}$ 는 각각 $\mathrm{d}$ 축 및 $\mathrm{q}$ 축의 목표 전류이고 $\hat{L}_{s}, \hat{\Lambda}_{m}$ 는 각각 인덕턴스와 쇄교 자속의 공칭 파라미터이다.

이상적으로 시스템 파라미터에 불확실성이 없는 경우 (즉, $L_{s}=\hat{L}_{s}, R_{s}=\hat{R}_{s}, \Lambda_{m}=\hat{\Lambda}_{m}$ ), 제어 입력 (4)-(5)를 사용 하면 식 (1)-(2)에서 $\omega_{r}$ 이 곱해진 항이 소거되므로 각각의 폐루프 전달함수가 식 (6)과 같이 되도록 PI 제어기를 결정 할 수 있다. 이 때, 제어 이득은 $K_{p}=\omega_{c} \hat{L}_{s}, K_{i}=\omega_{c} \hat{R}_{s}$ 이고 시 스템이 원하는 응답을 갖도록 $\omega_{c}$ 를 선택한다.

$$
\frac{i_{d}^{r}(s)}{i_{d}^{r^{*}}(s)}=\frac{\omega_{c}}{s+\omega_{c}}, \frac{i_{q}^{r}(s)}{i_{q}^{r^{*}}(s)}=\frac{\omega_{c}}{s+\omega_{c}}
$$

위와 같은 방법으로 폐루프 시스템을 1 차 시스템으로 제 어하면 토크 $\tau_{e}$ 의 응답을 간단하게 결정할 수 있고 2 차 시 스템에서 나타나는 오버슈트 등에 의한 토크 리플을 고려하 지 않아도 되는 장점이 있다.

\section{3 제안하는 전향 보상 제어기}

제어 식 (4)-(5)의 전향 보상 방법은 속도 측정과 전동기 파라미터에 불확실성이 존재할 경우 기대했던 제어 성능을 얻기 어렵다. 본 논문에서는 속도 정보 없이 파라미터 불확
실성으로 인한 성능 변화를 줄이기 위해 시스템의 불확실성 을 추정할 수 있는 PI 관측기를 전향 보상기로 적용한다. 제 안하는 방법은 공칭 시스템에 설계된 PI 제어기에 독립적으 로 설계할 수 있어 편리하고, 공칭 시스템의 역-전달함수를 이용하는 외란 관측기법[14,15] 등에 비해 제어기의 저차이 고 설계가 간단하여 보다 실제적인 제어 방법이다.

관측기 설계를 위해 식 (1)-(2)를 아래 식과 같이 다시 표현한다.

$$
\begin{aligned}
& v_{d}^{r}=\hat{R}_{s} i_{d}^{r}+\hat{L}_{s} \frac{d i_{d}^{r}}{d t}-f_{d}^{r} \\
& v_{q}^{r}=\hat{R}_{s} i_{q}^{r}+\hat{L}_{s} \frac{d i_{q}^{r}}{d t}-f_{q}^{r}
\end{aligned}
$$

위 식에서 불확실성 $f_{d}^{r}, f_{q}^{r}$ 은 식 (1)-(2)에서 각속도 $\omega_{r}$ 이 곱 해진 항뿐만 아니라 전동기의 공칭 파라미터 값과 실제 값 이 다른 경우 $\left(L_{s} \neq \hat{L}_{s}, R_{s} \neq \hat{R}_{s}, \Lambda_{m} \neq \hat{\Lambda}_{m}\right)$ 에 대한 그 차이를 포함한다. 즉, 아래 식과 같이 표현할 수 있다.

$$
\begin{aligned}
& f_{d}^{r}=-\Delta R_{s} i_{d}^{r}-\Delta L_{s} \frac{d i_{d}^{r}}{d t}+\omega_{r} L_{s} i_{q}^{r} \\
& f_{q}^{r}=-\Delta R_{s} i_{q}^{r}-\Delta L_{s} \frac{d i_{q}^{r}}{d t}-\omega_{r}\left(L_{s} i_{q}^{r}+\Lambda_{m}\right)
\end{aligned}
$$

이 때, $\Delta L_{s}=L_{s}-\hat{L}_{s}, \Delta R_{s}=R_{s}-\hat{R}_{s}$ 이다.

식 (9)의 불확실성 $\left(f_{d}^{r}, f_{q}^{r}\right)$ 추정을 위해 본 논문에서는 불 확실성의 변화가 전류에 비해 느리다고 가정하고 이를 상수 로 고려한다. 즉, $\left[\begin{array}{ll}f_{d}^{r} & f_{q}^{r}\end{array}\right]^{T}=\left[\begin{array}{ll}0 & 0\end{array}\right]^{T}$ 로 둔다. 편의상 아래 식 (10)과 같이 변수들을 정의하면 시스템 (7)-(8)을 식 (11)의 상태 공간 방정식으로 다시 쓸 수 있다.

$$
\begin{gathered}
x=\left[\begin{array}{l}
i_{d}^{r} \\
i_{q}^{r}
\end{array}\right], f=\left[\begin{array}{l}
f_{d}^{r} \\
f_{q}^{r}
\end{array}\right], u=\left[\begin{array}{l}
v_{d}^{r} \\
v_{q}^{r}
\end{array}\right] \\
\dot{x}=A x+N f+H u \\
\dot{f}=0 \\
y=\left[\begin{array}{ll}
C & 0
\end{array}\right]\left[\begin{array}{l}
x \\
f
\end{array}\right] \\
A=\left[\begin{array}{cc}
\hat{R}_{s} & 0 \\
\hat{L}_{s} & \hat{R}_{s} \\
0 & -\frac{\hat{R}_{s}}{\hat{L}_{s}}
\end{array}\right], N=H=\left[\begin{array}{ll}
\frac{1}{\hat{L}_{s}} & 0 \\
0 & \frac{1}{\hat{L}_{s}}
\end{array}\right], C=\left[\begin{array}{ll}
1 & 0 \\
0 & 1
\end{array}\right]
\end{gathered}
$$

시스템 (11)은 관측 가능하므로 식 (13)과 같이 관측기를 설계할 수 있다. 관측기의 오차 시스템은 식 (14)와 같다.

$$
\begin{aligned}
& \dot{\hat{x}}=A \hat{x}+N \hat{f}+H u+G_{1}(y-\hat{y}) \\
& \dot{\hat{f}}=G_{2}(y-\hat{y}) \\
& {\left[\begin{array}{c}
\dot{e}_{x} \\
\dot{e}_{f}
\end{array}\right]=\left[\begin{array}{cc}
A-G_{1} C & N \\
-G_{2} C & 0
\end{array}\right]\left[\begin{array}{l}
e_{x} \\
e_{w}
\end{array}\right]}
\end{aligned}
$$

단, $\left[\begin{array}{ll}e_{x} & e_{f}\end{array}\right]^{\mathrm{T}}=\left[\begin{array}{ll}x-\hat{x} & f-\hat{f}\end{array}\right]^{\mathrm{T}}$ 이고, 관측기 이득 $G_{1}, G_{2}$ 은 오 차 시스템 (14)가 안정하도록 설계한다.

식 (4)-(5)에서 전향 보상에 사용한 정보 $\left(\omega_{r} \hat{L}_{s} i_{q}^{r}\right.$, $\omega_{r} \hat{L}_{s} i_{d}^{r}+\omega_{r} \hat{\Lambda}_{m}$ ) 대신 관측기 (13)에서 추정된 $\hat{f}_{d}^{r}$ 과 $\hat{f}_{q}^{r}$ 을 적 용하면 제안하는 제어기를 다음과 같이 얻을 수 있다.

$$
\begin{aligned}
& v_{d}^{r}=K_{p} e_{1}+K_{i} \int e_{1} d t-\hat{f}_{d}^{r} \\
& v_{q}^{r}=K_{p} e_{2}+K_{i} \int e_{2} d t-\hat{f}_{q}^{r}
\end{aligned}
$$


다음 장에서는 모의실험과 실제 실험을 통해 제안하는 방 법의 성능을 시험한다. 식 (11)에서 상수로 가정한 불확실성 이 실제로 상수 값을 갖지 않더라도 제안하는 기법이 전류 제어에 효과적으로 쓰일 수 있음을 확인할 수 있다.

\section{3. 실험 결과}

제어 대상 전동기는 Emerson사의 630W SPMSM이고 그 공칭 파라미터는 표 1 과 같다[16]. 단, 마찰 계수 $B$ 는 데이 터에 포함되어 있지 않아 임의의 작은 값으로 선정하였다.

전류 제어 성능을 시험하기 위한 기준 입력은 구형파를 사용하였다. 무부하 상태에서 $\mathrm{q}$ 축 전류를 $i_{q}^{r^{*}}= \pm 1.5[\mathrm{~A}]$ 로 제 어하고, $\mathrm{d}$ 축 전류는 $i_{d}^{r^{*}}=0[\mathrm{~A}]$ 으로 제어한다. PI 제어기의 이 득 결정은 표 1 을 사용하였다. 폐루프 시스템의 cut-off 주 파수가 $\omega_{c}=100$ 일 때 $K_{p}=2.895, K_{i}=1225$ 이고, 관측기 이득 은 두 극점이 모두 -2000 에 위치하도록 결정하였다.

표 1 Unimotor 95UMA300의 공칭 파라미터

Table 1 Nominal parameters of Unimotor 95UMA300

\begin{tabular}{|c|c|c|c|}
\hline$\hat{R}_{s}$ & $12.25[\Omega]$ & $\hat{L}_{s}$ & $0.02895[\mathrm{H}]$ \\
\hline$\hat{\Lambda}_{m}$ & 0.2514 & $P$ & 3 \\
\hline$\hat{J}$ & $0.14 \times 10^{-3}\left[\mathrm{~kg}-\mathrm{m}^{2}\right]$ & $\hat{B}$ & $0.01 \times 10^{-3}[\mathrm{Nm} /(\mathrm{rad} / \mathrm{s})]$ \\
\hline
\end{tabular}

\section{1 모의실험}

실제 실험에 앞서 모의실험을 통해 제안된 방법의 추정 성능을 시험한다. 강인성 시험을 위해 전동기의 실제 저항 과 인덕턴스 값을 $R_{s}=2 \hat{R}_{s}, L_{s}=2 \hat{L}_{s}$ 로 설정하였다.

먼저 전향 보상의 유무에 따른 제어 성능 비교를 위해 PI 제어기만 사용한 제어 입력을 (불확실성이 없는) 공칭 시스 템에 인가하고 그 결과를 그림 1-2에 함께 나타내었다. 그 림에서 전향 보상기가 없는 경우 정상 상태 오차가 발생하 고 제어 성능이 만족스럽지 못함을 확인할 수 있다.

불확실성이 존재할 때 제안된 전향 보상기 (15)와 식 (4)-(5)의 전류 제어 성능을 비교하여 그림 3과 그림 4에 나 타내었다. 다른 방법과의 비교를 위해 외란 관측기 (DOB) $[14,15]$ 를 사용한 결과를 함께 나타내었다. 이 때, $\mathrm{Q}^{-}$ 필터는 시정수 $\tau=0.001$ 인 아래와 같은 2 차 저역 필터를 사 용하였다. 이는 제안된 방법에 비해 알고리즘이 복잡하고 $\mathrm{Q}$-필터 설계 등 제어기를 실제로 구현할 때 고려해야할 변 수가 증가한다.

$$
Q(s)=\frac{1}{(\tau s+1)^{2}}
$$

그림 3과 4에서 불확실성으로 인해 제어 식 (4)-(5)는 성 능이 저하된 반면 제안하는 방법 (15)로 얻은 결과는 불확 실성에도 불구하고 공칭 파라미터를 이용한 그림 1 및 2 의 경우와 거의 흡사함을 관찰할 수 있다. 이를 확인하기 위해 그림 1 과 2 의 경우와 불확실성이 있는 그림 3 과 4 의 차이를 그림 5와 6에 나타내었다. 이를 통해 제안된 방법이 실제 전류 제어 문제에 적용될 수 있음을 예상할 수 있다.

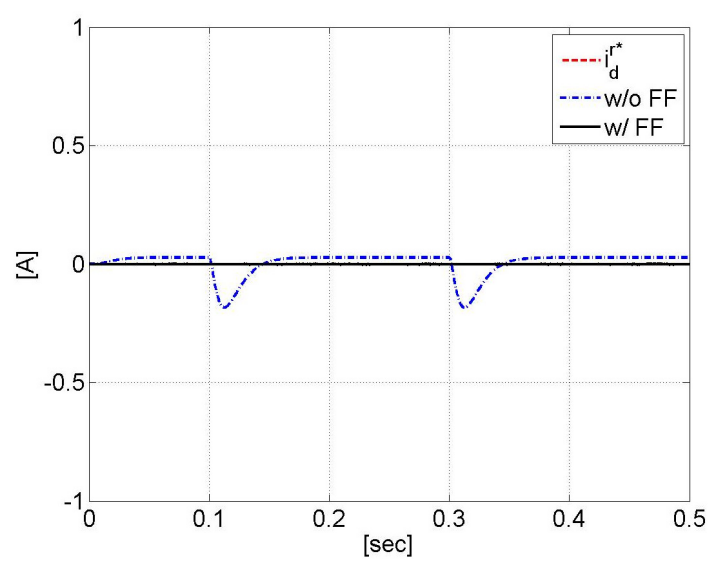

그림 1 전향보상 효과: d축 전류 $\left(i_{d}^{r^{*}}=0[\mathrm{~A}]\right)$

Fig. 1 Effect of Feedforward Compensation: $d$-axis current $\left(i_{d}^{* *}=0[\mathrm{~A}]\right)$

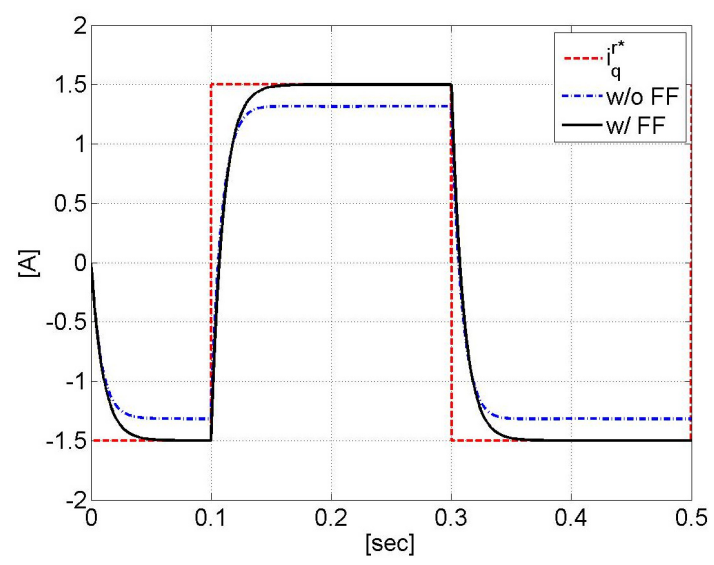

그림 2 전향보상 효과: q축 전류 $\left(i_{q}^{r^{*}}= \pm 1.5[\mathrm{~A}]\right)$

Fig. 2 Effect of Feedforward Compensation: q-axis current $\left(i_{q}^{r^{*}}= \pm 1.5[\mathrm{~A}]\right)$

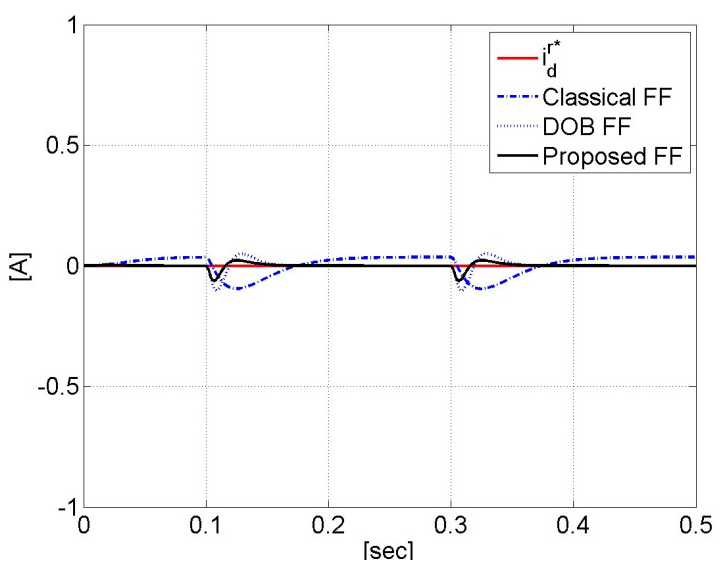

그림 $3 \mathrm{~d}$ 축 전류 비교 $\left(i_{d}^{r^{*}}=0[\mathrm{~A}]\right)$

Fig. 3 Comparison of $\mathrm{d}$-axis current $\left(i_{d}^{r^{*}}=0[\mathrm{~A}]\right)$ 


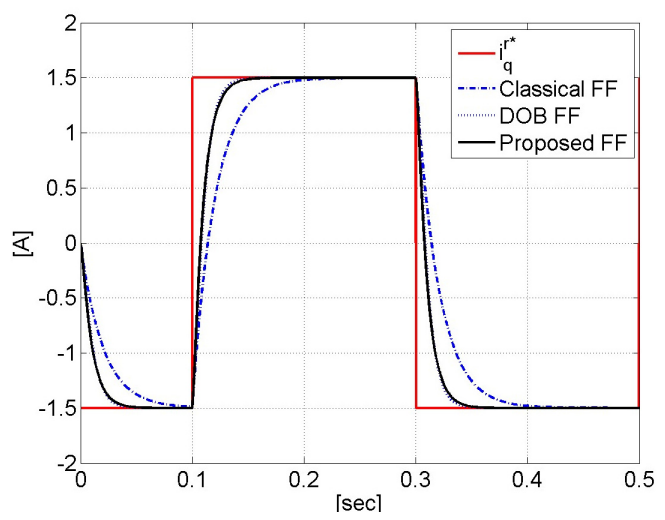

그림 $4 \mathrm{q}$ 축 전류 제어 비교 $\left(i_{q}^{r^{*}}= \pm 1.5[\mathrm{~A}]\right)$

Fig. 4 Comparison of $\mathrm{q}$-axis current $\left(i_{q}^{r^{*}}= \pm 1.5[\mathrm{~A}]\right)$

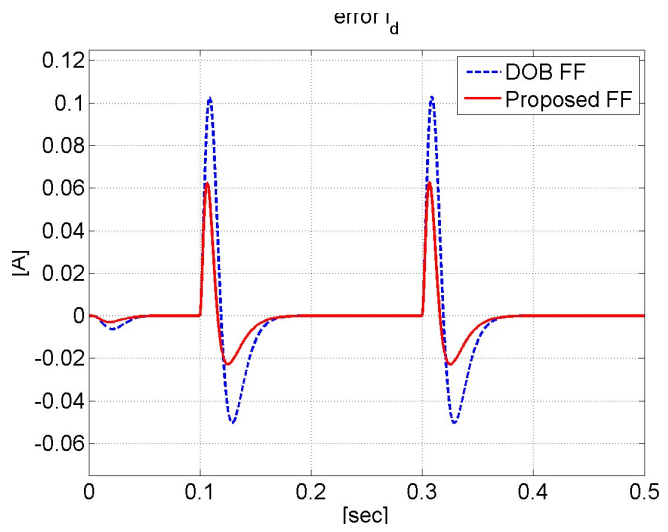

그림 5 전향보상 성능: $d$ 축 전류

Fig. 5 Feedforward Compensation Error: d-axis current

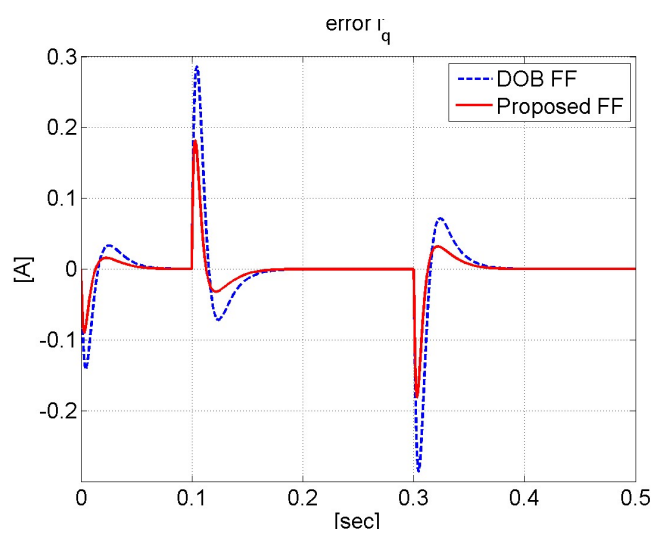

그림 6 전향보상 성능: $\mathrm{q}$ 축 전류

Fig. 6 Feedforward Compensation Error: q-axis current

제안된 관측기의 불확실성 추정 성능 분석을 위해 $\mathrm{q}$ 축 전 류로 인해 변화되는 전동기의 각속도 $\left(\omega_{r}\right)$ 를 그림 7 에 나타 내었다. 그림 8 과 9 에서는 실제 불확실성 $\left(f_{d}^{r}, f_{q}^{r}\right)$ 및 관측기 를 사용하여 추정한 결과 $\left(\hat{f}_{d}^{r}, \hat{f}_{q}^{r}\right)$ 를 함께 도시하였다.

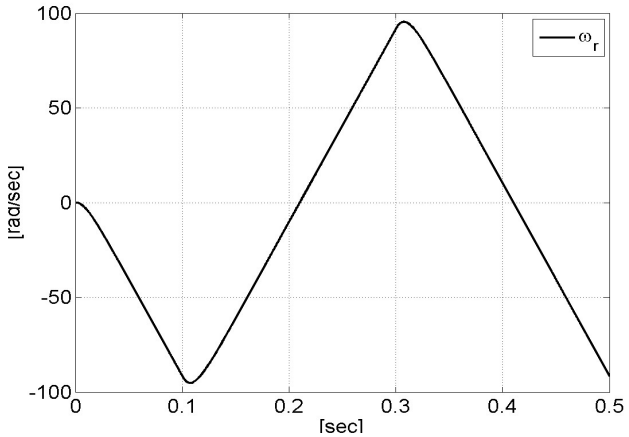

그림 $7 \mathrm{SPMSM}$ 의 전기 각속도 $\left(\omega_{r}\right)$

Fig. 7 Electrical angular velocity of $\operatorname{SPMSM}\left(\omega_{r}\right)$

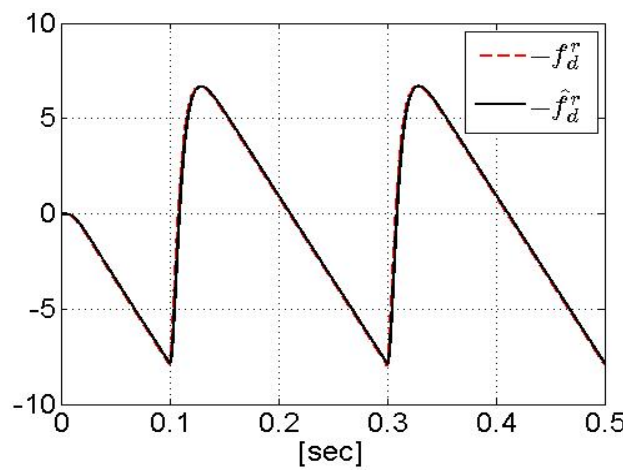

그림 $8 \mathrm{~d}$ 축 불확실성 추정 $\left(-f_{d}^{r}\right)$

Fig. $8 \mathrm{~d}$-axis uncertainty estimation $\left(-f_{d}^{r}\right)$

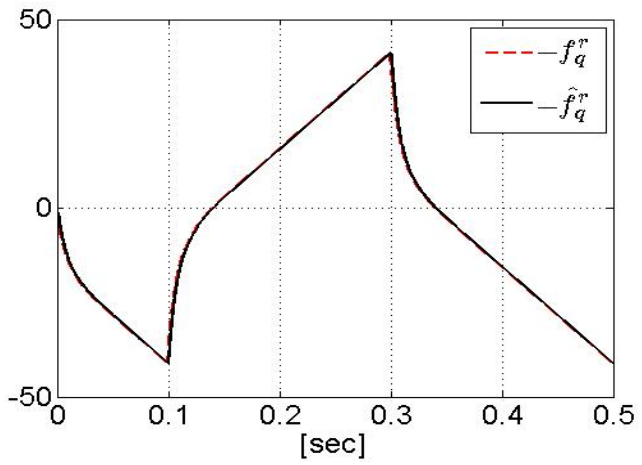

그림 $9 \mathrm{q}$ 축 불확실성 추정 $\left(-f_{q}^{r}\right)$

Fig. 9 q-axis uncertainty estimation $\left(-f_{q}^{r}\right)$

비록 식 (11)의 가정과 달리 불확실성이 상수 값을 갖지 않지만 전류의 동특성에 비해 불확실성의 변화가 충분히 느 려 관측기 (13)이 잘 동작함을 확인할 수 있다. 또한, 추정 오차가 다소 존재하더라도 함께 사용하는 PI 제어기에 의해 제어 목표가 달성됨을 알 수 있다.

\section{$3.2600 \mathrm{~W}$ 급 SPMSM 전류 제어 실험}

제안된 제어 방법의 실제 적용 가능성을 확인하기 위해 그림 10 과 같이 부하 시험기(유도 전동기)와 제어 대상 전 동기(Unimotor 95UMA300)로 구성된 실험 장치를 사용하여 
성능 실험을 진행하였다. 제어 알고리즘은 TI사의 $\mathrm{DSP}$ 인 TMS320F28335를 사용하여 디지털 제어기로 구현하였으며 이 때 샘플링 주파수는 $10 \mathrm{kHz}$ 이다.

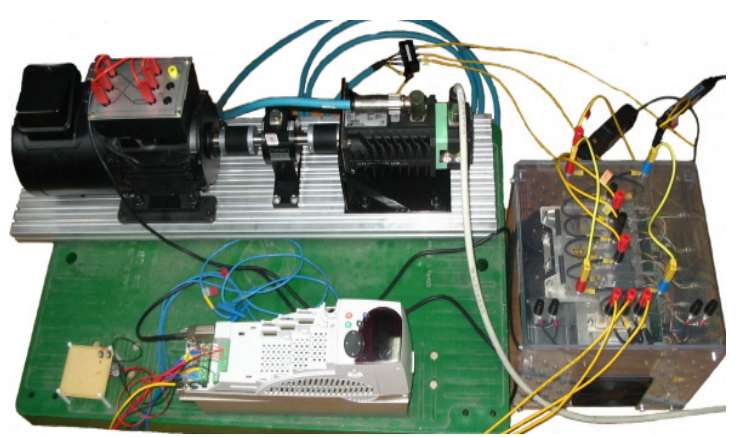

그림 10 Unimotor 95UMA300의 실험 장치

Fig. 10 Experiment set of Unimotor 95UMA300

모의실험과 같은 방법으로 진행한 실험 결과를 그림 11 그림 14에 나타내었다. 그림 11은 전향 보상기를 사용하지 않은 PI 제어기를 통해 얻은 결과이며 그림 12는 식 (4)-(5) 의 제어기를 사용한 경우이다. 그림 13 은 제안된 제어기 (15)를 사용한 결과이며 그림 14 에서 그림 12 와 그림 13 의 결과를 비교하였다. 불확실성으로 인해 공칭 파라미터를 이 용한 결과에서 성능 저하가 발생하였음을 알 수 있다. 실험 결과가 모의실험 결과와 유사하므로 제안된 전향 보상 방법 을 사용하는 경우 불확실성에 대한 전류 제어 성능이 개선 됨을 확인 할 수 있다.

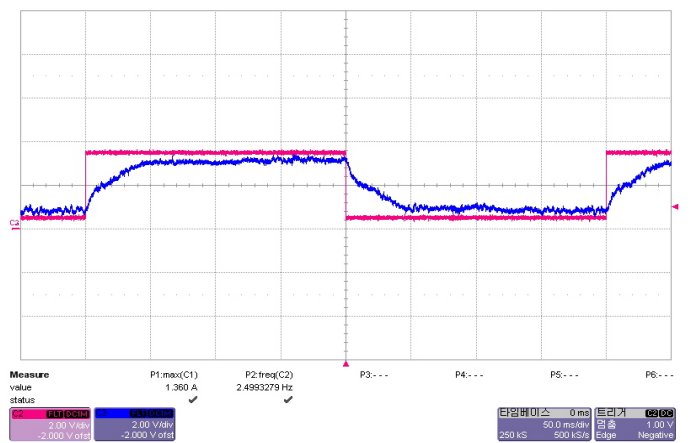

그림 11 전향 보상을 하지 않은 실험 결과

Fig. 11 Results without feed-forward compensation

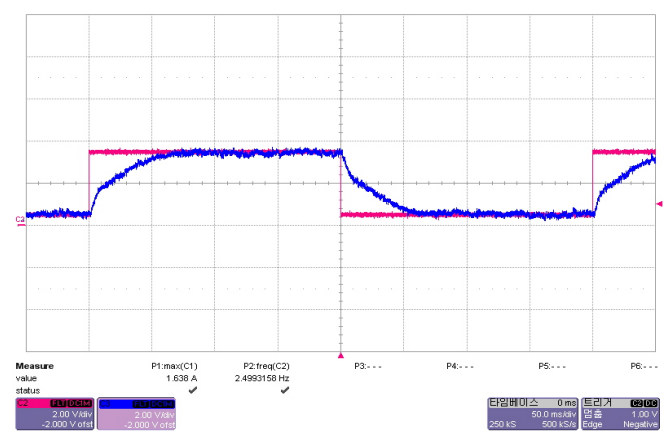

그림 12 고전적인 전향보상 기법을 사용한 실험 결과

Fig. 12 Results using classical FF compensation

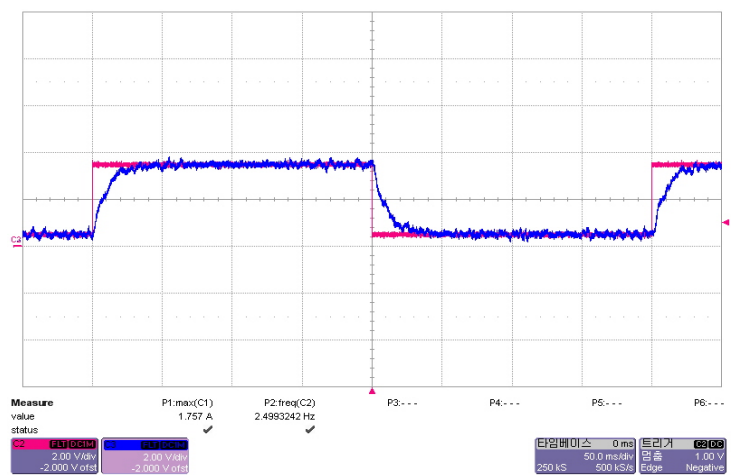

그림 13 제안된 전향보상 기법을 사용한 실험 결과

Fig. 13 Results using proposed FF compensation

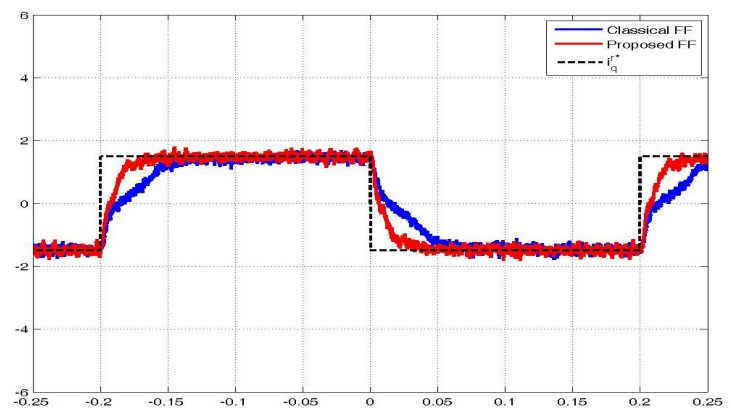

그림 14 전류 제어 성능 비교

Fig. 14 Comparison of current control performance

\section{4. 결 론}

본 논문은 표면부착형 영구자석 동기기(SPMSM)의 전류 제어기로 주로 사용되고 있는 PI 제어기와 전향 보상기를 결합한 제어기의 성능 개선에 대해 다루었다. 속도 정보를 사용하지 않고 불확실성을 추정하기 위해 $\mathrm{PI}$ 관측기를 이용 한 전향 보상 방법을 제안하였으며 상수로 가정한 불확실성 의 추정 성능을 모의실험과 실제 실험을 통해 확인하였다.

제안된 방법의 실제 시스템에 대한 적용 가능성을 시험하 기 위해 $600 \mathrm{~W}$ 급 하드웨어 장비로 실험을 실시하였으며, 모 의실험과 유사한 실험 결과를 통해 전류 제어 성능이 개선 됨을 확인하였다. 제안된 제어기는 구조가 간단하고 디지털 제어기로도 쉽게 구현할 수 있어 실제적인 전동기 제어 문 제에 다양하게 활용될 수 있다. 향후 전류 센서의 측정 잡 음에 대한 보상 및 전동기 속도 제어 문제를 위한 다양한 실험 연구가 진행될 계획이다.

\section{감사의 글}

이 연구에 참여한 연구자는 『2단계 $\mathrm{BK} 21$ 사업』의 지원비를 받았음. 본 결과물은 지식경제부의 에너지자 원인력양성사업의 지원으로 명지대학교 분산전원복합 운용연구센터를 통해 수행되었습니다. 


\section{참 고 문 헌}

[1] S. Bolognani, L. Zigliotto, E. Bertotto, "Commissioning of Electromechanical Conversion Models for High Dynamic PMSM Drives", IEEE Trans. on Industrial Electronics, vol. 57, no. 3, pp. 986-993, 2010.

[2] S. Carriere, S. Caux, M. Fadel, "Optimised Speed Control in State Space for PMSM Direct Drives", IET Electric Power Appl., vol. 4, no. 3, pp. 158-168, 2010.

[3] 이광운, "저 분해능 위치센서를 갖는 표면부착형 영구자석 동기전동기의 전류센서 없는 단위 전류 당 최대 토크 제 어” 전력전자학회 논문지, vol. 13, no. 3, pp. 204-210, 2009.

[4] 박철훈, 손영수, 함상용, 김병인, 윤동원, “코깅토크 측정 장치 직접구동용 영구자석 동기전동기의 정밀속도제어”, 한국정밀공학회지, vol. 26, no. 5, pp. 79-86, 2009.

[5] D.M. Brod, D.W. Novotny, "Current Control of VSI-PWM Inverters", IEEE Trans. on Industry Applications, vol. IA-21, no. 4, pp. 562-570, 1985.

[6] M. Kurishige, T. Kifuku, "Static Steering Control System for Electric Power Steering", Mitsubishi Electric Advance, vol. 94, pp. 18-20, June, 2001.

[7] X. Chen, T. Yang, X. Chen, and K. Zhou, "A Generic Model-Based Advanced Control of Electric Power-Assisted Steering Systems", IEEE Trans. Control Systems Technology, vol. 16, no. 6, pp. 1289-1300, 2008.

[8] Y.A.-R.I Mohamed, E.F. El-Saadany, "A Current Control Scheme With an Adaptive Internal Model for Torque Ripple Minimization and Robust Current Regulation in PMSM Drive Systems", IEEE Trans. on Energy Conversion, vol. 23, no. 1, pp. 92-100, 2008.

[9] 설승기, 전기기기 제어론, 홍릉과학출판사, 2007.

[10] K.Y. Cho, J.D. Bae, S.K. Chung, M.J. Youn, "Torque Harmonics Minimization in Permanent Magnet Synchronous Motor with Back EMF Estimation", IEE Proc.-Electr. Power Appl., vol. 141, no. 6, pp. 323-330, 1994.

[11] S. Lerdudomsak, S. Doki, S. Okuma, "A Novel Current Control System for PMSM Considering Effects from Inverter in Overmodulation Range", Proc. of PEDS'07, pp. 794-800, 2007.

[12] S. Beale, B. Shafai, "Robust Control System Design with a Proportional Integral Observer", Int. J. Control, vol. 50, no. 1, pp. 97-111, 1989.

[13] B.K. Bose, Modern Power Electronics and AC Drives, Prentice Hall, 2002.
[14] P. Mattavelli, "An Imploved Deadbeat Control for UPS Using Disturbance Observers“, IEEE Trans. on Industrial Electronics, vol. 52 no. 1, pp. 206-212, 2005.

[15] 김성종, 손영익, 정유석, "단상 $\mathrm{DC} / \mathrm{AC}$ 인버터 시스템의 부하변동을 고려한 외란 관측기 기반 제어기”, 전자공학회 논문지, vol. 44-SC, no. 4, pp. 21-26, July, 2007.

[16] www.controltechniques.com, www.emerson.com
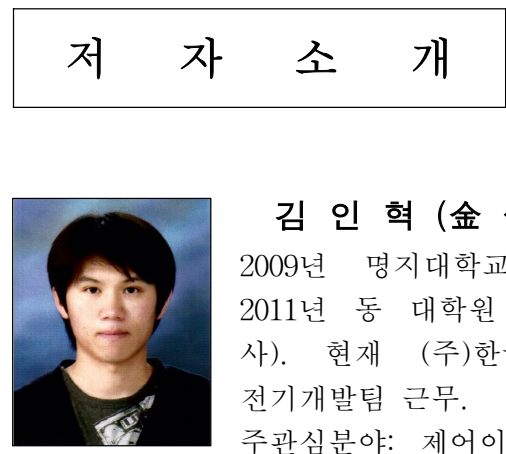

김 인 혁 (金 仁 䚂)

2009년 명지대학교 전기공학과 졸업. 2011년 동 대학원 전기공학과 졸업(석 사). 현재 (주)한국미쓰비시엘리베이터 전기개발팀 근무.

주관심분야: 제어이론 및 적용, 디지털 제어기, 임베디드 시스템,

Tel : 032-585-5282

E-mail : ihkim@k-mec.co.kr

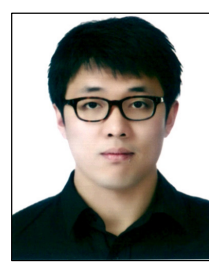

\section{최 대 식 (崔大 植)}

2010년 명지대학교 전기공학과 졸업. 2010년 현재 명지대학교 전기공학과 석 사과정.

주관심분야: 제어이론 및 적용, 모터 제 어, 전력 변환기

Tel : 031-330-6358

E-mail : choitek@mju.ac.kr

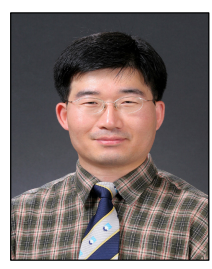

\section{손 영 익 (孫 瑛 翼)}

1995년 서울대학교 전기공학과 졸업. 2002년 동 대학원 전기 - 컴퓨터공학부 졸업(공박). 2007년 2008년 Cornell University Visiting Scholar. 2003년 현 재 명지대학교 전기공학과 부교수.

Tel : 031-330-6358

E-mail : sonyi@mju.ac.kr 